\title{
Tissue Factor Expression by Endothelial Cells in Sickle Cell Anemia
}

\author{
Anna Solovey, Lizhen Gui, Nigel S. Key, and Robert P. Hebbel \\ Department of Medicine, University of Minnesota, Minneapolis, Minnesota 55455
}

\begin{abstract}
The role of the vascular endothelium in activation of the coagulation system, a fundamental homeostatic mechanism of mammalian biology, is uncertain because there is little evidence indicating that endothelial cells in vivo express tissue factor (TF), the system's triggering mechanism. As a surrogate for vessel wall endothelium, we examined circulating endothelial cells (CEC) from normals and patients with sickle cell anemia, a disease associated with activation of coagulation. We find that sickle CEC abnormally express TF antigen (expressed as percent CEC that are TF-positive), with $66 \pm 13 \%$ positive in sickle patients in steady-state, $83 \pm 19 \%$ positive in sickle patients presenting with acute vasoocclusive episodes, and only $10 \pm 13 \%$ positive in normal controls. Repeated samplings confirmed this impression that TF expression is greater when sickle patients develop acute vasoocclusive episodes. Sickle CEC are also positive for TF mRNA, with excellent concurrence between antigen and mRNA expression. The TF expressed on the antigen-positive CEC is functional, as demonstrated by a binding assay for Factor VIIa and a chromogenic assay sensitive to generation of Factor Xa. By establishing that endothelial cells in vivo can express TF, these data imply that the vast endothelial surface area does provide an important pathophysiologic trigger for coagulation activation. (J. Clin. Invest. 1998. 101:1899-1904.) Key words: endothelium • sickle $\bullet$ tissue factor $\bullet$ coagulation $\bullet$ hemostasis
\end{abstract}

\section{Introduction}

As one of the fundamental homeostatic mechanisms of mammalian biology, the blood coagulation system establishes a delicate balance between the procoagulant and anticoagulant functions of blood and the vessel wall, thereby guarding against excesses in either direction and normally preventing unwanted hemorrhage or thrombosis. System components include a cascade of interacting proteases, accelerating steps that provide amplification, a variety of balancing feedback controls, and, most importantly, tightly regulated activation (1). The latter is provided by controlled expression of tissue factor

Address correspondence to Dr. Robert P. Hebbel, University of Minnesota Hospitals and Clinics, Box 480, Harvard St. at E. River Rd., Minneapolis, MN 55455. Phone: 612-624-6104; FAX: 612-625-6919; E-mail: hebbe001@maroon.tc.umn.edu

Received for publication 3 October 1997 and accepted in revised form 24 February 1998.

J. Clin. Invest.

(C) The American Society for Clinical Investigation, Inc. 0021-9738/98/05/1899/06 \$2.00

Volume 101, Number 9, May 1998, 1899-1904

http://www.jci.org
$(\mathrm{TF}),{ }^{1}$ a transmembrane protein that provides for calciumdependent binding of coagulation Factor VII and its activated form, Factor VIIa $(2,3)$. When bound to TF, the activity of Factor VIIa towards its target substrates, zymogen Factors IX and $\mathrm{X}$, is enhanced by orders of magnitude over that of unbound Factor VIIa (4). The resulting explosive generation of Factor Xa (activated Factor X) establishes the amplification of activity that culminates in clot formation. Consistent with the role of TF as the triggering mechanism that stops bleeding, cells that surround blood vessels (e.g., fibroblasts and smooth muscle cells) constitutively express TF, thus establishing a procoagulant envelope beyond the endothelial barrier that comprises the blood-tissue interface (5).

However, in contrast to this clear role in physiologic hemostasis, the participation of these extravascular sites of TF expression in pathologic thrombotic states is less clear. Hence, it is suspected that cells normally exposed to the blood may trigger coagulation activation in such states, and attention has focused on blood monocytes. While these do not constitutively express TF, by means of transcriptional regulation they readily express it in response to a great variety of biological stimuli (2, 3). Examples of thrombotic diatheses in which monocyte TF is implicated include sepsis (6), thrombotic risk after hip surgery (7), and coronary artery disease (8). As a consequence of the hypothesized role of monocytes and the concept of a strictly extravascular procoagulant envelope, a role for endothelial expression of TF in vivo has generally not been accepted. On the other hand, under artificial conditions in vitro endothelial cells can be induced to express TF (see Discussion). Thus, whether or not the vascular endothelium in vivo expresses TF comprises an extant controversy in our understanding of this fundamental biologic process.

To test the possibility that endothelial TF expression might occur in vivo in the context of vascular pathobiology, we elected to study subjects with sickle cell anemia (homozygotes for the mutant hemoglobin S) because these patients seem to be in a state of constant hemostatic perturbation (9). Among the lines of evidence supporting this notion are observation of abnormal levels of prothrombin fragment F 1.2 and D-dimer $(9,10)$, documentation of accelerated Factor VII turnover (11), detection of increased thrombin-antithrombin complexes (11), and discovery of abnormal expression of TF on blood monocytes (12). Hence, we hypothesized that the vascular endothelium in sickle cell anemia would exhibit a procoagulant phenotype, with expression of functional TF.

\section{Methods}

Subjects. Volunteer study subjects included 10 normal donors, 12 patients with sickle cell anemia (homozygous for hemoglobin S) who were sampled a total of 25 times, 4 subjects with sickle cell trait (heterozygous for hemoglobin $\mathrm{S}$ ), and 2 donors with other hemolytic anemias persisting despite surgical splenectomy. In accordance with our

1. Abbreviations used in this paper: $\mathrm{CEC}$, circulating endothelial cells; $\mathrm{TF}$, tissue factor. 
institutional review board, donors were adults and gave informed consent, except for two minors with sickle cell anemia who gave assent after parental permission was obtained. Venous blood was processed immediately for study.

Antibodies and reagents. Murine mAb $\mathrm{P} 1 \mathrm{H} 12$ was used to harvest circulating endothelial cells (CEC) and to identify blood cells as being endothelial. As previously described (13), testing of multiple cell lines and tissues established that $\mathrm{P} 1 \mathrm{H} 12$ reacts only with endothelial cells. Moreover, the circulating cells that are positive for P1H12 are also positive for both von Willebrand factor and thrombomodulin, and they express flt-1 and KDR, receptors for the endothelialspecific mitogen VEGF. A polyclonal blocking antibody to TF was kindly provided by Dr. Ron Bach (Minneapolis Veterans Administration Medical Center, Minneapolis, MN). Recombinant Factors VIIa and $\mathrm{X}$ and $\mathrm{mAbs}$ to TF and to Factor VII/VIIa (specific recognition epitopes unknown) were obtained from American Diagnostica Inc. (Greenwich, CT).

CEC enrichment. For assay of TF antigen expression, we fixed cells immediately after venipuncture by adding $0.25 \%$ paraformaldehyde to whole blood, incubating for $10 \mathrm{~min}$, and washing three times with PBS. Samples were then restored to four times the initial blood volume with HBSS/EDTA/BSA (HBSS with $1 \mathrm{mM}$ EDTA and $0.5 \%$ BSA). As previously described in detail (13), a CEC-enriched preparation was then obtained using immunomagnetic beads (Dynal, Oslo, Norway) coated with P1H12. To do this, we admixed $4 \times 10^{6}$ beads for each milliliter of original blood, incubated for $1 \mathrm{~h}$ with gentle agitation, and collected $\mathrm{CEC} /$ bead rosettes using a magnetic concentrator (Dynal). Then, the CEC/bead rosettes were affixed to coverslips, washed with PBS, and blocked with $3 \%$ BSA/PBS for 30 min before subsequent immunofluorescence studies.

Fluorescence microscopy. Examination involved dual staining using FITC-, Cy3-, or biotin-labeled $\mathrm{P} 1 \mathrm{H} 12$ with a second primary antibody (to TF or Factor VII/VIIa) with appropriate labeled secondary antibodies, in the fashion described previously (13). Positive controls for expression of TF or binding of Factor VIIa were provided by cultured fibroblasts (which constitutively express TF), as well as by cultured endothelial cells stimulated to express TF by in vitro exposure to endotoxin. Negative controls were provided by the TF-negative white blood cells contaminating CEC preparations and by unstimulated cultured endothelial cells.

Live/dead assay. A fluorescent kit (Molecular Probes, Eugene, OR) that distinguishes live cells (green cytoplasmic fluorescence) from dead cells (red nuclear fluorescence) was used, exactly as described previously (13), in conjunction with dual immunofluorescence examination to determine whether CEC expressing TF were alive or dead.

In situ hybridization. This was performed on paraformaldehydefixed cytospin preparations of CEC using established methods (14). Plasmid pHTF containing TF cDNA, kindly provided by Dr. William Konigsberg (Yale University, New Haven, CT), was digested with AvaI/HindIII and inserted into plasmid pGem4z (Promega Corp., Madison, WI) to generate pHTF-Gem4. Linearized pHTF-Gem4 digested with AvaI (antisense probe) and pHTF digested with HindIII (sense probe) were used as templates for in vitro transcription. FITC and digoxigenin labeling of RNA probes was performed using Ambion (Austin, TX) and Boehringer Mannheim (Indianapolis, IN) kits, respectively, according to the manufacturers' directions. Digoxigenin-labeled RNA was detected immunologically with labeled antidigoxigenin antibody (Boehringer Mannheim). Cultured fibroblasts and endotoxin-stimulated endothelial cells were used as positive controls, and unstimulated endothelial cells were used as negative controls; cells were tested with both sense and antisense probes. Immunological staining with $\mathrm{P} 1 \mathrm{H} 12$ and/or anti-TF antibodies followed in situ hybridization and was performed as above.

Functional assays. For assays of TF function, we carried out CEC enrichment before fixation. In this case, the fresh whole blood was diluted fourfold with HBSS/EDTA/BSA before admixture with immunomagnetic beads and collection of $\mathrm{CEC} /$ bead rosettes, as above.
Then, CEC preparations were split, with one aliquot being treated with EDTA to remove any preexisting Factor VII/VIIa. After this, samples were applied to coverslips, washed with PBS, fixed with $4 \%$ paraformaldehyde for $10 \mathrm{~min}$ at room temperature, washed again, and finally blocked with $3 \%$ BSA, as above.

For functional assay, recombinant Factor VIIa was added (10 nM for $1.5 \mathrm{~h}$ ) in the presence of $8 \mathrm{mM}$ calcium, and cells were tested for Factor VIIa binding by immunofluorescence microscopy. To further test for TF function, recombinant Factor X (100 nM for $30 \mathrm{~min}$ at $37^{\circ} \mathrm{C}$ ) was also added to $\mathrm{CEC}$, followed by a 5 -min exposure to $1 \mu \mathrm{M}$ fluorescein-di-( $p$-guanidinobenzoate) which becomes fluorescent when activated by Factor Xa. Samples were taken at various steps in this process for preparation and microscopic examination.

For functional assays, positive controls were provided by cultured fibroblasts and endotoxin-activated cultured endothelial cells. Negative controls were provided by unstimulated endothelial cells. Control experiments (not shown) documented that TF-positive cells (endotoxin-stimulated human umbilical vein endothelial cells, fibroblasts, and CEC) were unable to exhibit Factor $\mathrm{X}$ activation (i.e., convert fluorescein-di-[ $p$-guanidinobenzoate] to its fluorescent derivative) when incubated with either Factor VIIa alone or Factor X alone (data not shown), verifying that this chromogenic substrate is activated only by Factor Xa and not by Factor VIIa or zymogen Factor X.

Since CEC enrichment was carried out before fixation for these functional studies, in control experiments (not shown) we tested our handling procedures for ability to activate endothelial cells during processing. For eight sickle samples, we tested TF antigen expression when the CEC were fixed after the enrichment procedure. For these samples, we found that $71 \pm 17 \%$ of CEC were TF-positive, a value not significantly different from the value for those CEC fixed before enrichment (78 $\pm 19 \%$ ). Additionally, we carried cultured human umbilical vein endothelial cells through the entire enrichment procedure and verified that they remained TF-negative. Thus, we are confident that our processing procedures do not artificially activate CEC to express TF.

\section{Results}

Due to the inaccessibility of vessel-wall endothelium for study, we developed methods that allow phenotypic analysis of CEC, which should be an acceptable surrogate because they would be subjected to the same blood-borne, endothelial-activating influences as vessel-wall endothelium. Our approach to this required addressing two problems: identification of CEC and their harvesting for study. As described recently, we solved these problems by developing an endothelial-specific antibody (P1H12) and by using immunomagnetic beads coated with $\mathrm{P} 1 \mathrm{H} 12$ to obtain CEC-enriched preparations from fresh whole blood (13). This was necessitated because of the very small number of CEC (2.6 \pm 1.6 per $\mathrm{ml}$ of blood for normals, with 10 fold higher numbers in sickle donors) (13). Two-thirds of the $\mathrm{CEC}$ in sickle patients are alive and three-quarters mark as being microvascular in origin (13).

TF antigen. Using immunofluorescence microscopy for dual detection of $\mathrm{P} 1 \mathrm{H} 12$ positivity and TF antigen (Fig. 1, $A$ and $B$ ), we found $\mathrm{TF}$ positive $\mathrm{CEC}$ in blood from sickle cell anemia patients. The percentage of CEC that expressed TF antigen in these patients $(78 \pm 19 \%)$ was much greater than in normals $(10 \pm 13 \%)$, with no overlap between the two groups (Table I). Values were within the normal range for sickle trait donors (heterozygotes for hemoglobin S) and even for control donors with hemolytic states persisting after surgical splenectomy, the best control for sickle cell anemia which is characterized by a chronic hemolytic state and functional asplenia (Table I). 

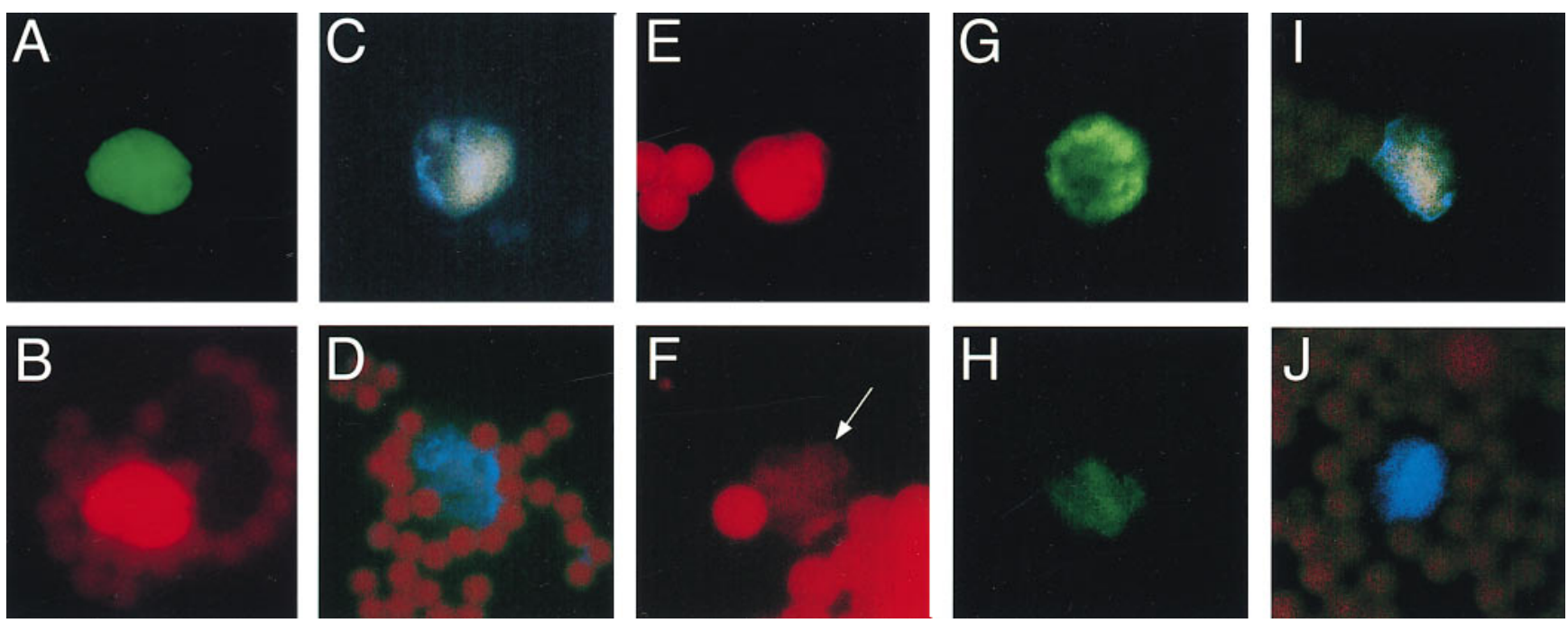

Figure 1. Examples of CEC analysis using fluorescence techniques. All panels show CEC obtained from sickle patients. Several of the panels include not only a CEC but also several of the beads used for CEC preparation; the beads also accumulate color because they are coated with antibody to mouse IgG. $(A$ and $B)$ A single CEC is shown to be positive for P1H12 $(A)$ and TF antigen $(B)$. $(C$ and $D)$ In $C$, a cell that is P1H12 positive (blue) shows hybridization with antisense probe to TF mRNA (yellow). In $D$, a CEC (blue) in the control aliquot shows no hybridization with the control sense probe. ( $E$ and $F$ ) A CEC stains positively with an antibody to Factor VII/VIIa $(E)$, and binding is abolished with a blocking antibody to TF ( $F$, in which the faint CEC is indicated by an arrow). ( $G$ and $H$ ) A CEC incubated with Factors VIIa and X exhibits Factor X activation, as detected by a chromogenic substrate sensitive to Factor Xa $(G)$. Activity is abolished by a blocking antibody to TF $(H)$. $(I$ and $J)$ A CEC identified by $\mathrm{P} 1 \mathrm{H} 12$ positivity (not shown) exhibits simultaneous expression of TF antigen (blue) and TF mRNA which is green-yellow in color because the probe is yellow $(I)$. In contrast, the CEC in $J$ is positive for TF antigen (blue) but is negative for hybridization with the sense control probe (yellow).

To demonstrate the variability in intensity of TF positivity, we illustrate in Fig. 2 the TF antigen staining of all $10 \mathrm{CEC}$ identified (by their $\mathrm{P} 1 \mathrm{H} 12$ positivity, which is not shown) in a single sample from one of the sickle donors. Three of the cells are negative for TF; of the seven positive cells, six have an intense signal while one is less strongly positive. This is a representative example, since in all samples studied, greater than three-fourths of TF-positive CEC were intensely positive, as illustrated in Fig. $1 \mathrm{~B}$.

As shown in Fig. 3, we observed TF positivity in CEC from the sickle patients not only at the onset of acute painful episodes (caused by vascular occlusion) but also during their chronic steady-state (i.e., remote by at least 1 mo from acute events of any kind). However, the degree of CEC positivity for TF was significantly higher in the acute group than in the chronic group (Fig. 2): $83 \pm 19 \%(n=17)$ vs. $66 \pm 13 \%(n=8)$,

Table I. Expression of TF Antigen on CEC

\begin{tabular}{lccc}
\hline & & \multicolumn{2}{c}{ CEC positive for TF } \\
\cline { 3 - 4 } & & Mean \pm SD & Range \\
\hline & $n$ & $\%$ & $\%$ \\
Normal (HbAA) & 10 & $10 \pm 13$ & $0-33$ \\
Sickle cell anemia (HbSS) & 25 & $78 \pm 19 *$ & $50-100$ \\
Sickle cell trait (HbAS) & 5 & $11 \pm 15$ & $0-28$ \\
Splenectomized HbAA hemolytic controls & 2 & 29 & 25,33 \\
& & & \\
\end{tabular}

$* P<0.001$ compared to normal value. respectively $(P=0.023)$. Notably, every sample that exhibited $100 \%$ positivity for TF expression was found in the acute patient group (Fig. 2). In addition, repeated testing for CEC positivity as four patients moved between steady-state and acute
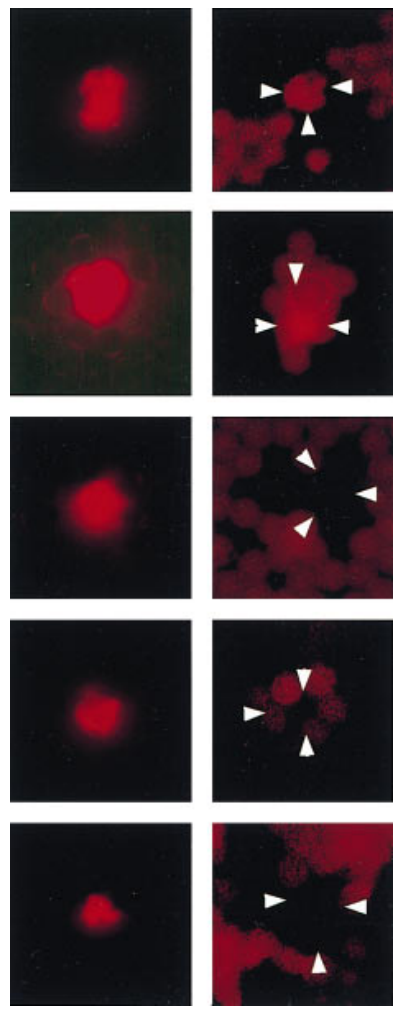

Figure 2. All 10 CEC from one sickle sample (identified by their P1H12 positivity [not shown]) are shown after staining for TF antigen expression. Three CEC are negative (bottom right). Seven are positive, six intensely positive, and one only very weakly (top right). Arrows show edges of the CEC as defined by P1H12 staining. Faint round shapes seen in some panels are the magnetic beads used for CEC enrichment. 


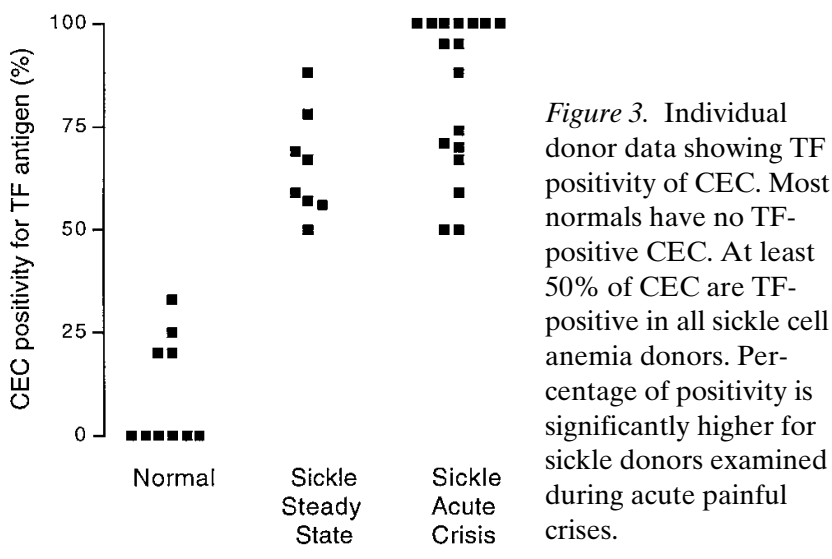

crisis further supports the conclusion that CEC positivity tends to be greater at onset of acute painful episodes (Fig. 4).

Inspection of individual donor data (Fig. 3) reveals that expression of CEC TF is above $0 \%$ for an occasional normal subject. The rare TF-positive CEC in normal blood has a large impact on the percent positivity because the total number of CEC is so small in normals; that is, the values of 20,25 , and $33 \%$ positivity in normals correspond to a single positive cell out of 5, 4, and 3 total CEC, respectively. Importantly, the percentage of TF positivity was precisely the same for the live and dead CEC ( $r=0.999, P<0.001)$, indicating that induction of TF expression is unrelated to, and has preceded, CEC death in vivo. The correspondence between TF antigen expression and other measures of CEC TF is summarized in Table II and presented in detail in the following sections.

$T F m R N A$. To exclude the possibility that CEC positivity for $\mathrm{TF}$ is simply due to adsorption of soluble TF from plasma, we confirmed by in situ hybridization that many sickle CEC
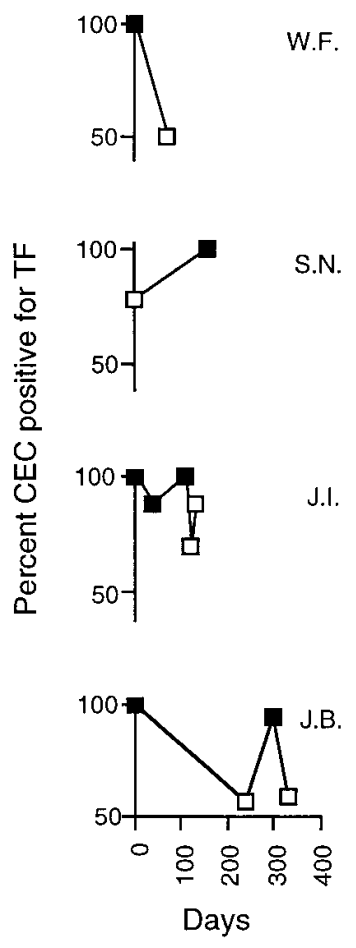

Figure 4. Repeated study of sickle patients. The CEC positivity for TF was tested for four sickle patients when the they were in steady-state (open squares) and at presentation of acute painful crisis (closed squares).
Table II. Correspondence Between Measures of TF Expression by $C E C$

\begin{tabular}{lc}
\hline & CEC positive \\
\hline & $\%$ \\
TF antigen and TF mRNA $(n=5)$ & $82 \pm 18$ \\
TF antigen & $78 \pm 17$ \\
TF mRNA & $73 \pm 31$ \\
TF antigen and Factor VIIa binding $(n=8)$ & $74 \pm 23$ \\
TF antigen & \\
Factor VIIa binding & $97 \pm 3$ \\
TF antigen and Factor Xa generation $(n=3)$ & $84 \pm 7$ \\
TF antigen & \\
Factor Xa generation & \\
\hline
\end{tabular}

Data shown as mean \pm SD for separate experiments comparing TF antigen expression with TF mRNA, Factor VIIa binding, or Factor Xa generation.

actually express TF mRNA (Fig. 1, $C$ and $D$ ). Signal was positive only using the antisense probe (Fig. $1 C$ ), not using the control sense probe (Fig. $1 D$ ). The overall correspondence between expression of TF antigen and mRNA was excellent: in five sickle samples, $82 \pm 18 \%$ of CEC expressed antigen and $78 \pm 17 \%$ expressed mRNA $(r=0.892, P=0.042)$. In three of these samples, we examined individual CEC for colocalization of TF antigen and TF mRNA (Fig. $1, I$ and $J$ ), finding that of all CEC positive for either marker, $94 \pm 10 \%$ were positive for both and only $6 \pm 10 \%$ were positive singly for one or the other.

TF function. Since biological relevance derives from function, we applied two tests for CEC expression of functional TF. First, we tested the ability of CEC to bind Factor VIIa (Fig. 1, $E$ and $F$ ). For eight sickle samples tested in this manner, $73 \pm 31 \%$ of CEC expressed TF antigen, and $64 \pm 22 \%$ stained directly with antibody to Factor VII/VIIa (i.e., even without addition of Factor VIIa in vitro), suggesting that the CEC that are positive for TF antigen actually carry Factor VII/VIIa on their surface while in the blood stream. (Although there is no direct proof that this represents specific binding to TF, it probably does since not all CEC were positive for Factor VII/VIIa, and there is a good correspondence between percentages of CEC that are positive for TF and for Factor VII/VIIa.) After treatment of these CEC with EDTA, $0 \%$ stained with antibody to Factor VII/VIIa. (Since after EDTA treatment and washing these cells were blocked with $3 \%$ BSA before testing with the anti-Factor VII/VIIa antibody, this result incidentally excludes the possibility that bovine Factor VII/VIIa contaminating BSA would contribute to our detection of Factor VII/ VIIa.) Then, upon readdition of reagent Factor VIIa in vitro, $74 \pm 23 \%$ were found to stain for Factor VII/VIIa, corresponding perfectly to the percentage of CEC originally positive for staining for TF antigen (Figure $1 E$ ). Binding of Factor VIIa was prevented by chelation of calcium and by a blocking antibody to TF (Fig. $1 F$ ).

We also tested CEC for their ability to exhibit conversion of Factor X to Factor Xa (Fig. 1, $G$ and $H$ ). To CEC stripped of any preexisting factors using EDTA, we added back calcium, Factor VIIa, and Factor X in the presence of a chromogenic substrate that becomes fluorescent if it is activated by Factor 
Xa. In three patients having $97 \pm 3 \%$ of CEC positive for TF antigen, we found $84 \pm 7 \%$ of CEC to be positive for generation of Factor Xa in this fashion (Figure $1 G$ ). CEC Factor Xa activity was fully inhibited by substitution of EDTA for calcium, by omission of Factor VIIa, and by pretreatment of CEC with a blocking antibody to TF (Fig. $1 H$ ).

\section{Discussion}

To address the controversy over whether or not the vascular endothelium exhibits pathophysiologic expression of TF in vivo, we have studied CEC. Because these cells tend to be alive and responsive to phenotype-altering stimuli (13), and would be subjected to the same modifying influences as vascular-wall endothelium, we believe they are a suitable surrogate for study. Accepting that the normal, quiescent endothelium should not express TF in the absence of biologic stimulation $(5,15)$, we wished to examine CEC in an appropriate patient population; that is, in the context of a pathophysiologic disturbance of the coagulation system. Thus, we selected for study patients with sickle cell anemia, a disease which involves virtually constant hemostatic activation and for which there is circumstantial evidence for abnormal TF activity in vivo (9-12). By documenting the abnormal expression of functional TF on CEC in these patients, our findings argue that the vascularwall endothelium can, indeed, participate in the TF-dependent triggering of the blood coagulation system. The endothelial surface area is vast and estimated to comprise $10^{12}$ endothelial cells in the adult human (16). By comparison, there are only $\sim 10^{10}$ blood monocytes. Thus, it seems highly likely that endothelial cell expression of TF can play a pathobiologic role in the tightly regulated activation of coagulation.

In fact, our data are consistent with the known ability of cultured endothelial cells to express TF in vitro in response to a great variety of stimuli, ${ }^{2}$ which has comprised the strongest previous argument that they might do so in vivo. Only in a few very special circumstances has in vivo endothelial TF expression been previously described: in the splenic vasculature of baboons during sepsis (17), rarely in placental vessels (18), and sometimes in breast cancer vessels (19). While one histochemical assessment reported that endothelium overlying atherosclerotic plaques does express TF (20), another using in situ hybridization found an absence of expression by the overlying endothelium (15). It may be that the general paucity of data supporting endothelial TF expression in tissues is related to suboptimal selection of patients for study. That is, TF is not likely to be detected in tissue endothelium in the absence of hemostatic activation state. In this regard, an unexpected finding is that a rare CEC was found to be positive for TF in a minority of normal donors. The significance of this is unknown at this time. It also is of interest that CEC isolated from sickle blood already carry Factor VIIa on their surface, since it suggests that vessel-wall endothelium may provide a sink for re-

2. Agents that have been reported to induce TF expression by endothelial cells in vitro include: endotoxin; thrombin; IL-1; TNF; homocysteine; E-selectin/ICAM linkage; oxidized LDL; monocytes; infection with HSV-1, Staphylococcus, HIV, or rickettsia; phorbol; cycloheximide; complement fixation; and oxygen radicals. The endotoxin effect is augmented by ionophore A23187, monocytes with aggregated $\operatorname{IgG}$, and by hypoxia via platelet-activating factor. moval of this factor from plasma. This could explain why systemic activation of coagulation in sickle patients (12) and in patients with disseminated intravascular coagulation (21) is paradoxically associated with lowered levels of blood Factor VIIa.

The implications of our data for sickle cell anemia itself are substantial. They help explain earlier data that implied abnormal TF activity in these patients $(9,11)$. The positivity of CEC for TF even in the steady-state patients (with no overlap between these and normals), along with a parallel abnormal expression of TF on blood monocytes (12), provides the most direct evidence to date that the hemostatic system of these patients is activated even when they appear to be clinically well and suggests that they are in a condition of perpetual procoagulant tone. A previous study of 45 sickle patients and 50 normals showed that every sickle patient, regardless of acute versus chronic status, had elevated levels of thrombin/antithrombin complexes in their plasma (11), and another study reported elevated levels of D-dimer in 23 of 25 sickle patients in steady-state and in all 21 patients in acute crises (10). Confirming this, we recently found elevated D-dimer levels in each of 18 sickle patients from our own population (from which the participants in this study were drawn), for patients in both steady-state and acute crisis, and with no overlap with normal values (12).

In aggregate, these data justify enhanced suspicion that disordered hemostatic mechanisms play a role in the vascular complications of this disease. While the specific stimuli accounting for $\mathrm{TF}$ expression on sickle CEC are not identified, some known endothelial-stimulating substances are variably or even chronically elevated in plasma of sickle cell anemia patients, including IL-1, endotoxin, TNF, and thrombin $(9,11)$. These findings are consistent with the regulatory role the endothelium plays as a fundamental participant in various physiologic functions, including inflammation, vasoregulation, adhesion biology, and hemostasis.

\section{Acknowledgments}

We thank Elizabeth Wayner for making the P1H12-producing hybridoma line available to us, Ron Bach for providing the blocking antibody to human TF, William Konigsberg for providing the TF cDNA, and Steve Nelson and Jeanne Harkness for assistance in obtaining blood samples.

This work was supported by the National Institutes of Health (grants HL-55174 and HL-55219).

\section{References}

1. Furie, B., and B.C. Furie. 1988. The molecular basis of blood coagulation. Cell. 53:505-518.

2. Edgington, T.S., N. Mackman, K. Brand, and W. Ruf. 1991. The structural biology of expression and function of tissue factor. Thromb. Haemost. 66: 67-79.

3. Nemerson, Y. 1992. The tissue factor pathway of blood coagulation. Semin. Hematol. 29:170-176.

4. Ruf, W., A. Rehemtulla, and T.S. Edgington. 1991. Phospholipid-independent and -dependent interactions required for tissue factor receptor and cofactor function. J. Biol. Chem. 266:2158-2166.

5. Drake, T.A., J.H. Morrissey, and T.S. Edgington. 1989. Selective cellular expression of tissue factor in human tissues. Implications for disorders of hemostasis and thrombosis. Am. J. Pathol. 134:1087-1097.

6. Edgington, T.S., N. Mackman, S.T. Fan, and W. Ruf. 1992. Cellular immune and cytokine pathways resulting in tissue factor expression and relevance to septic shock. Nouv. Rev. Fr. Hematol. 34:S15-S27.

7. Nygaard, O.P., K. Unneberg, O. Reikeras, and B. Osterud. 1990. Throm- 
boplastin activity of blood monocytes after total hip replacement. Scand. J. Clin. Lab. Invest. 50:183-186.

8. Leatham, E.W., P.M. Bath, J.A. Tooze, and A.J. Camm. 1995. Increased monocyte tissue factor expression in coronary disease. Br. Heart J. 73:10-13.

9. Francis, R.B., Jr., and R.P. Hebbel. 1994. Hemostasis. In Sickle Cell Disease: Basic Principles and Clinical Practice. S.H. Embury, R.P. Hebbel, N. Mohandas, and M.H. Steinberg, editors. Raven Press, New York. 299-310.

10. Francis, R.B. 1989. Elevated fibrin D-dimer fragment in sickle cell anemia: evidence for activation of coagulation during the steady-state as well as in painful crisis. Haemostasis. 19:105-111.

11. Kurantsin-Mills, J., F.A. Ofosu, T.K. Safa, R.S. Siegel, and L.S. Lessin. 1992. Plasma factor VII and thrombin-antithrombin III levels indicate increased tissue factor activity in sickle cell patients. Br. J. Haematol. 81:539-544.

12. Key, N.S., L. Dandelet, S.C. Nelson, L. Styles, F. Kuypers, and R.R. Bach. 1998. Elevated whole blood tissue factor and depressed activated factor VII levels in patients with sickle cell anemia. Blood. In press.

13. Solovey, A., Y. Lin, P. Browne, S. Choong, E. Wayner, and R.P. Hebbel. 1997. Circulating activated endothelial cells in sickle cell anemia. N. Engl. J. Med. 337:1584-1590.

14. Panoskaltsis-Mortari, A., and R.P. Bucy. 1995. In situ hybridization with digoxigenin-labeled RNA probes: facts and artifacts. Biotechniques. 18:300-307.
15. Wilcox, J.N., K.M. Smith, S.M. Schwartz, and D. Gordon. 1989. Localization of tissue factor in the normal vessel-wall and in the atherosclerotic plaque. Proc. Natl. Acad. Sci. USA. 86:2839-2843.

16. Jaffe, E.A. 1987. Cell biology of endothelial cells. Hum. Pathol. 12:234-239.

17. Drake, T.A., J. Cheng, A. Chang, and F.B. Taylor, Jr. 1993. Expression of tissue factor, thrombomodulin, and E-selectin in baboons with lethal Escherichia coli sepsis. Am. J. Pathol. 142:1458-1470.

18. Faulk, W.P., C.A. Labarrere, and S.D. Carson. 1990. Tissue factor: identification and characterization of cell types in human placentae. Blood. 76:8696.

19. Contrino, J., G. Hair, D.L. Kreutzer, and F.R. Rickles. 1996. In situ detection of tissue factor in vascular endothelial cells: correlation with the malig nant phenotype of human breast disease. Nat. Med. 2:209-214.

20. Thiruvikraman, S.V., A. Guha, J. Roboz, M.B. Taubman, Y. Nemerson, and J.J. Fallon. 1996. In situ localization of tissue factor in human atherosclerotic plaques by binding of digoxigenin-labeled factors VIIa and X. Lab. Invest. 75:451-461.

21. Mesters, R.M., P.M. Mannucci, R. Coppola, T. Keller, H. Ostermann, and J. Kienast. 1996. Factor VIIa and antithrombin III activity during severe sepsis and septic shock in neutropenic patients. Blood. 88:881-886. 